\title{
Computer Modelling of Two-level Digital Frequency Synthesizer with Poisson Probability Distribution of Output Pulses
}

\author{
Jinfei Wang ${ }^{1}$, Krzysztof Przystupa ${ }^{2}$, Volodymyr Maksymovych ${ }^{3}$, Roman Stakhiv ${ }^{3}$, Orest Kochan ${ }^{3,4}$ \\ ${ }^{1}$ School of Mechanical Engineering, Northwestern Polytechnical University, 127 Youyi Ave. West, Xi'an 710072, China; \\ wjfwenxn@sina.com \\ ${ }^{2}$ Faculty of Mechanical Engineering, Technical University of Lublin, Lublin, Poland, k.przystupa@pollub.pl \\ ${ }^{3}$ Institute of Computer Technologies, Automation and Metrology, Lviv Polytechnic National University, Lviv, Ukraine, \\ volodymyr.maksymovych@gmail.com,ristakhiv@gmail.com \\ ${ }^{4}$ School of Computer Science, Hubei University of Technology, Wuhan, China, orestvk@gmail.com
}

\begin{abstract}
The article presents the modified structure of the two-level digital frequency synthesizer (TLDFS), which combines the properties of classical digital frequency synthesizers (DFS) and Poisson pulse sequence generators (PPSG). The analysis of the statistical characteristics of synthesizer output signal, obtained in computer modelling with the use of appropriate software, has been carried out, which allowed determining the effective range of values of its control codes. The proposed generators can be effectively used to simulate various natural and technical processes, in particular, to simulate the output signals of dosimetric detectors during the design, adjustment and testing of dosimetric devices.
\end{abstract}

Keywords: Digital frequency synthesizer, Poisson pulse sequence, statistical characteristics, computer modelling.

\section{INTRODUCTION}

Various measurement sensors [1] and systems [2] are used in industry, science [3], [4], and technology [5], [6]. All of them need metrological service [7]. That is why the market of metrological service constitutes a considerable share of GDP in developed countries [7], [8]. The progress of instruments and instrumentation lies in standardization [9], error elimination techniques [10] and increasing metrological reliability of measurement results [11], developing new metrological techniques [12], [13], developing new verification and calibrating techniques, including calibration in-situ [14]-[16]. This progress is called for, because proper measurement instruments and sensors lead to considerable gain in ecology [17], [18], economy [18], science [19], medicine [20] and other areas, therefore studies in the field of metrological service are relevant and topical [11], [15].

\section{StATE OF THE ART}

Digital frequency synthesizers (DFS) are widely used in various fields of technology. Their main advantages, compared with analog devices, are the accuracy of output signal, resistance to external factors, reliability and high performance [21]-[23]. The basis of their structures is a digital storage device (accumulative adder) and a comparison scheme, which is also the basis of DFS. The latter are a subset of CSM with the two-level output signals. The papers [24], [25] show the improved structures of DFS with the extended functional capabilities, in which the possibility of changing the output frequency controlling is implemented.

Simulations of many natural and technical processes which follow the Poisson probability distribution often use PPSGs as a tool. The implementation of the PPSGs can have different structures [26]-[28], these include the pseudorandom number generator (PRNG) as well as the comparison scheme [29].

The disadvantage of existing PPSGs is the inability to control the step of changing the average frequency of the output signal, which complicates their use in many applications. At the same time, existing digital frequency synthesizers allow this to be done, but they are not designed to generate an output signal that is corresponding to the Poisson distribution law. This work is aimed at solving these disadvantages.

The similarity of TLDFS and PPSG constructing principles allows combining them into one structure, in which the extended possibilities for managing the output signal frequency are combined with its formation according 
to the Poisson probability distribution. Such a frequency synthesizer can be used to simulate different processes, in particular, to simulate the output signals of the dosimetric detectors, because the activation of various types of extremist and terrorist organizations around the world puts on the agenda the problem of control over the movement of radioactive materials both within a country and through borders of countries, in particular strengthening control over their movement through sea ports and airports. This makes new requirements to increase the accuracy, speed and reliability of dosimetric measurements.

The researches given in this paper are a continuation of the studies initiated in [30].

\section{STRUCTURE SCHEMES OF TLDFS}

The TLDFS can be implemented on the basis of the accumulative adder (AA), which includes a combinational adder CA, a register Rg, and an element of logical multiplication (Fig.1.).

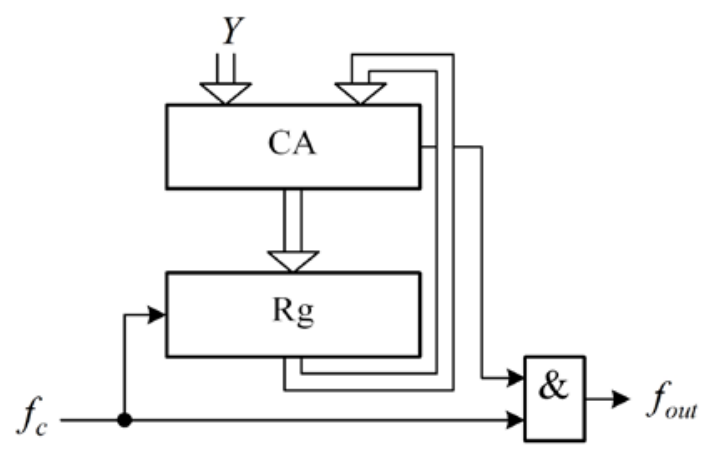

Fig.1. Structure scheme of the TLDFS.

The average value of the output signal frequency of such a device is determined by the equation

$$
f_{\text {out }} \cong \frac{Y}{N_{m}} f_{c}
$$

where $Y$ - the control code, $f_{c}-$ pulse generator frequency.

In case of the synthesizer implementation in the binary code: $N_{m}=2^{m}$ ( $m$ - the number of binary digits), and when it is implemented in the binary-decimal code $N_{m}=10^{m}$ ( $m$ - the number of decades).

An improved version of such a synthesizer is implemented using two combinational adders CA1 and CA2 [24]. It also includes the commutator $\mathrm{C}$ (Fig.2.).

Its main advantage is the ability to change the step of an output frequency grid. The average output frequency of the device is determined by the expression

$$
f_{\text {out }} \cong \frac{Y 1}{N_{m}-Y 2} f_{c}
$$

where: $Y 1$ and $Y 2$ - control codes.

The structures of the TLDFS output pulse sequences are given in Fig.1. and Fig.2. They are not uniform. Their nonuniformity is considered in [25] and the ways of its minimization are considered in [31].

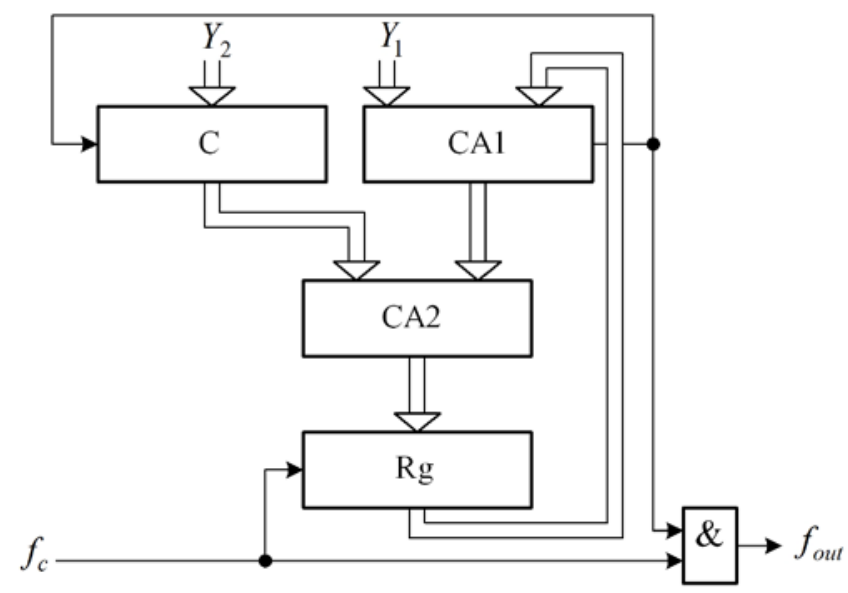

Fig.2. Structure scheme of the improved TLDFS.

\section{A. Structure schemes of the PRNG}

The well-known structure of the Poisson pulse sequence generator (PPSG) is presented in Fig.3. [29]. The average output frequency is described by (1). It consists of a pseudorandom number generator (PRNG), a comparison scheme CS, and an element of logical multiplication. Input clock pulses pass to the device output when the pseudorandom number at the PRNG output is smaller than the control code G.

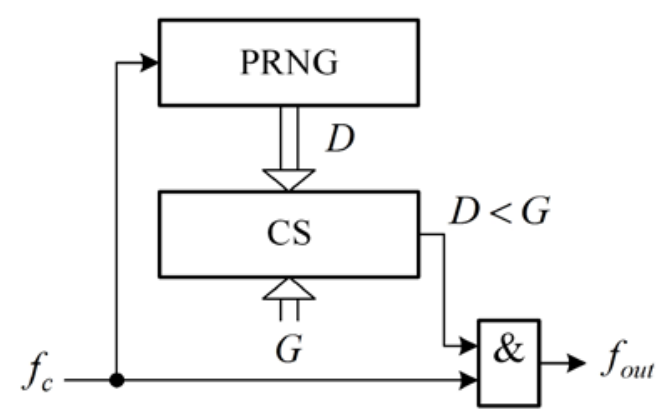

Fig.3. Structure scheme of the PRNG.

The average frequency of the generator output pulses equals

$$
f_{\text {out }} \cong \frac{G}{D_{\max }} f_{c}
$$

where $D_{\max }$ - the maximum possible value of the numbers at the PRNG output. 


\section{B. Structures schemes of the PPSG}

The purpose of this work is to construct a PPSG with the possibility of implementing an equation similar to (2). This will expand the functionality of the PPSG. On the other hand, such PPSG can be considered as TLDFS, whose output pulse sequence is subject of the Poisson probability distribution, which can be used, first of all, to simulate the output signals of the dosimetric detectors [29].

The proposed structure of the PPSG is given in Fig.4.

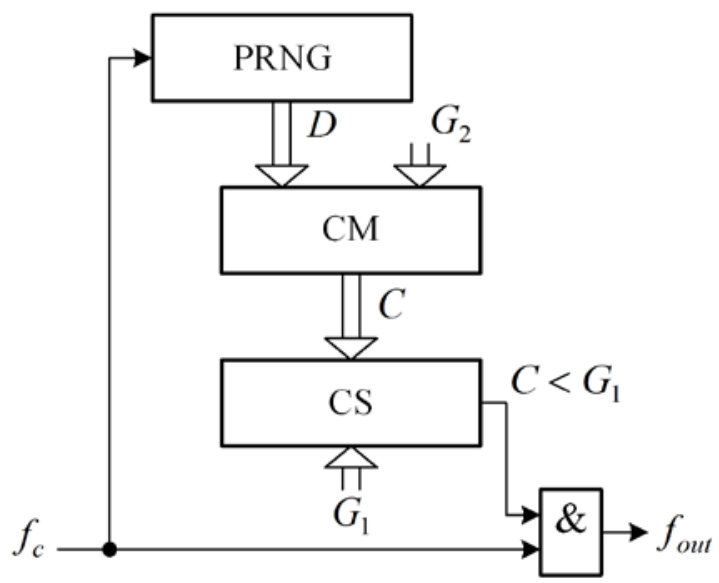

Fig.4. Structure scheme of the modified PPSG.

The structure of the generator additionally includes the code multiplier $\mathrm{CM}$, which generates the product of pseudorandom numbers $D$ by the value $\frac{D_{\max }-G_{2}}{D_{\max }}$.

The average value of the output frequency equals

$$
f_{\text {out }} \cong \frac{G_{1}}{D_{\max }-G_{2}} f_{c}
$$

where $G_{1}$ and $G_{2}$ are control codes.

The membership of a pulse sequence to the Poisson probability distribution depends on the statistical characteristics of pseudorandom number sequence at the output of the PRNG and can be verified using the methods described in [32], [33]. PRNG can be implemented in both hardware and software in different structures or algorithms [34], [35].

Some particular results of researches of the PPSG, constructed according to the structure shown in Fig.4. are presented below.

The dependences of the PPSG output frequency $f_{\text {out }}$ on the value of the control code $G_{1}$ with two different fixed values of the control code $G_{2}$ are shown in Fig.5. Dependencies are obtained as a result of simulation for the PPSG program implementation, including the software implementation of the PRNG using the function random (it is for Delphi), with $D_{\max }=1000$. Output frequency was determined by the number of output pulses in one second with the value of the clock frequency $f_{c}=1 \mathrm{kHz}$. Here the theoretical dependences of (4) are shown by the solid straight lines. The bounds that allow us to estimate the membership of the initial pulse sequence to the Poisson probability distribution are marked by the dashed lines. The mathematical expressions are given below (see (7) and (8)).

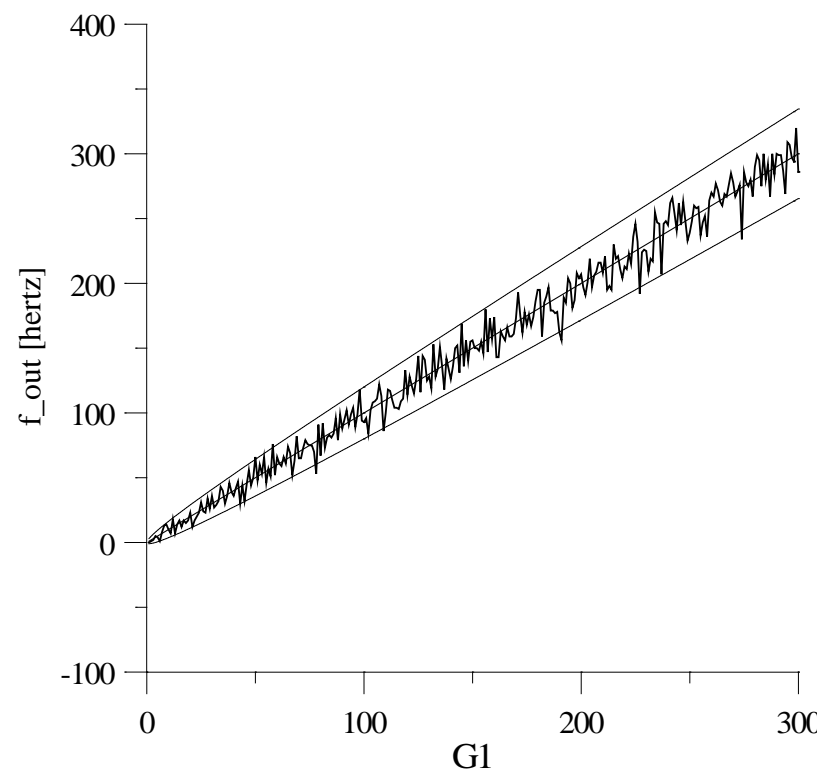

a)

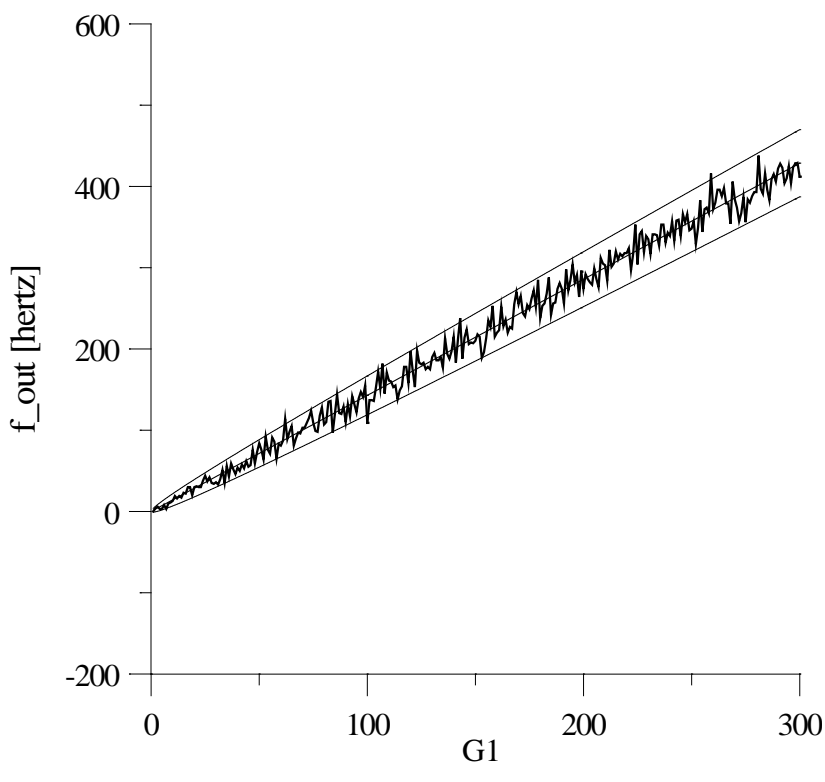

b)

Fig.5. The dependencies of the PPSG output frequency $f_{\text {out }}$ on the value of the control code $G_{1}$ : (a) - with fixed values of the control code $G_{2}=0$, (b) - with fixed values of the control code $G_{2}=300$. 


\section{ESTIMATION OF THE MEMBERSHIP OF THE OUTPUT PULSE} SEQUENCE TO THE POISSON PROBABILITY DISTRIBUTION

In [32], [33] the methods for estimating the PPSG output signal quality using the interval estimates and the Pearson criteria [36] for mathematical statistics are presented. They are sufficiently versatile, but at the same time tied to the estimation of generators constructed according to the structure shown in Fig.3. That is why, for the estimation of generator quality realized on the basis of the new structure
(Fig.4.), these methods require a certain correction, which is done in this work.

The flow of PPSG input pulses can be divided into $\mathrm{n}$ identical groups, each of which consists of $i_{\max }$ pulses (Fig.6.). We will denote the maximum number of groups $n_{\max }$ and the interval that corresponds to them $-T_{B}$. The groups of input pulses correspond to the groups of output pulses with the number of pulses $k_{1}, k_{2}, \ldots, k_{n_{\max }}$.

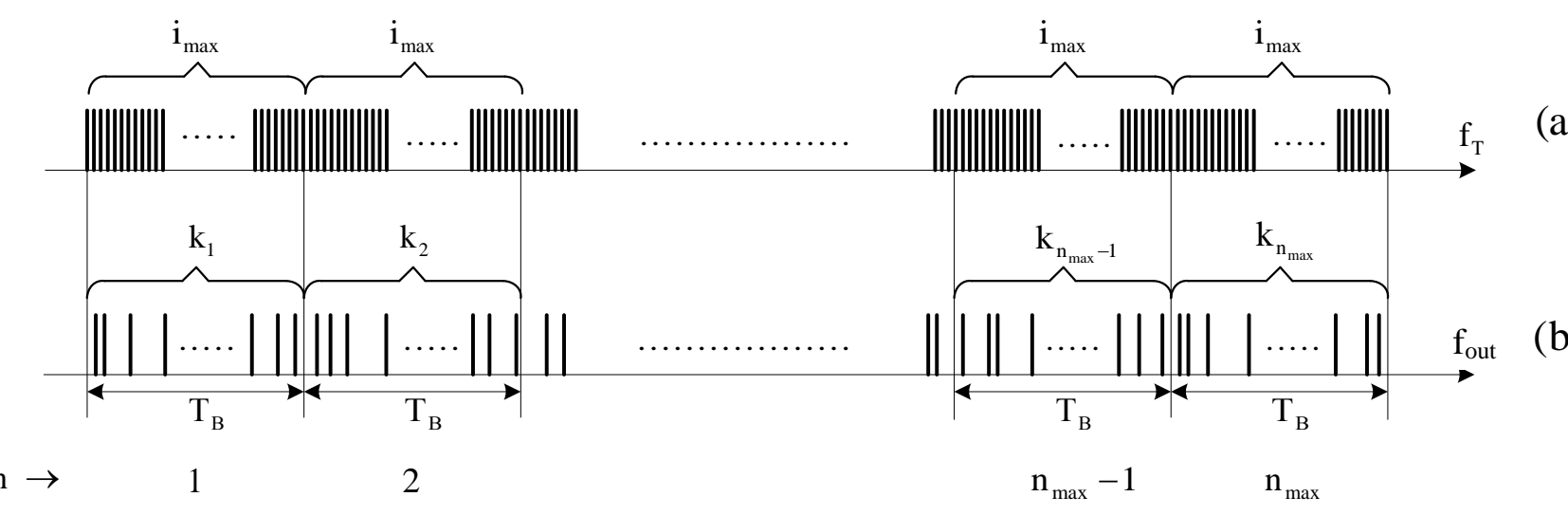

Fig.6. Divide of input and output pulse flows into groups: (a) - input pulses of PPSG, (b) - output pulses of PPSG.

Taking into account equation (4), the average number of output pulses in a group can be defined as follows:

$$
k_{C}=\frac{G_{1}}{D_{\max }-G_{2}} i_{\max }
$$

To research the statistical parameters of the PPSG output pulse sequence it is necessary:

- to select a mathematical device for estimating the statistical characteristics of the pulsed flow, taking into account its division into groups (Fig.6.);

- to select the value $i_{\max }$ and $n_{\max }$ depending on the number of digits (range of source code values) of the selected PRNG.

To estimate the statistical characteristics of the pulse flow in accordance with the Poisson probability distribution, one of the interval estimates can be used, according to which the number of pulses, during a fixed time $T_{B}$, with a probability $\mathrm{p}=0.95$, is within [36]

$$
k_{C}-2 \sqrt{k_{C}}<k<k_{C}+2 \sqrt{k_{C}}
$$

Accordingly, the limit values of the output signal average frequency are:

$$
\begin{aligned}
& f_{\text {out_h }}=f_{\text {out }}+2 \cdot \sqrt{f_{\text {out }}} \\
& f_{\text {out_l }}=f_{\text {out }}-2 \cdot \sqrt{f_{\text {out }}}
\end{aligned}
$$

where $f_{\text {out }}=k_{C} / T_{B}$ - the theoretical value of the output signal average frequency, which is determined by equation (4).

Graphs of dependency of functions $f_{\text {out_h }}$ and $f_{\text {out_l }}$ on control code $G_{1}$, which show that for this simulation experiment, the parameters of the generator output signal are close to the Poisson probability distribution, and they are presented by dashed lines in Fig.5.

In this paper we also propose the technique based on the classical method of testing the distribution hypothesis about the general population by the Poisson probability distribution by using the Pearson criterion (criterion $\chi^{2}$ ) [36]. It differs from the known [33] by the method of the value forming $i_{\max }$ that is necessary to take into account the peculiarities of the PPSG construction, which is studied (Fig.4.).

In accordance with this methodology:

- the nominal (theoretical) average value of numbers $k_{1}$, $k_{2}, \ldots, k_{n_{\max }}$ is fixed $-k_{c}$, regardless of the control code value $G$;

- the value $i_{\max }$ is variable, it depends on the value $G$ and is determined by the equation

$$
i_{\max }=\frac{D_{\max }-G_{2}}{G_{1}} k_{c}
$$




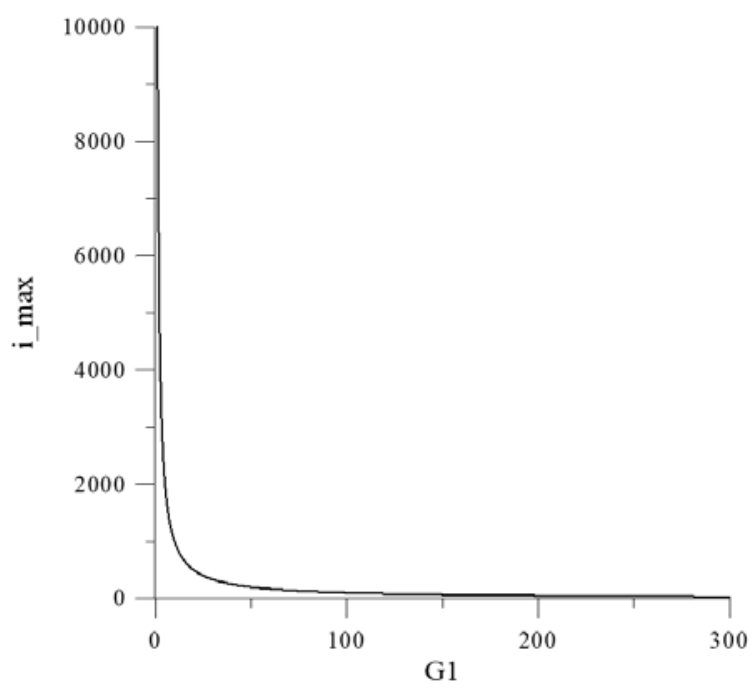

a)

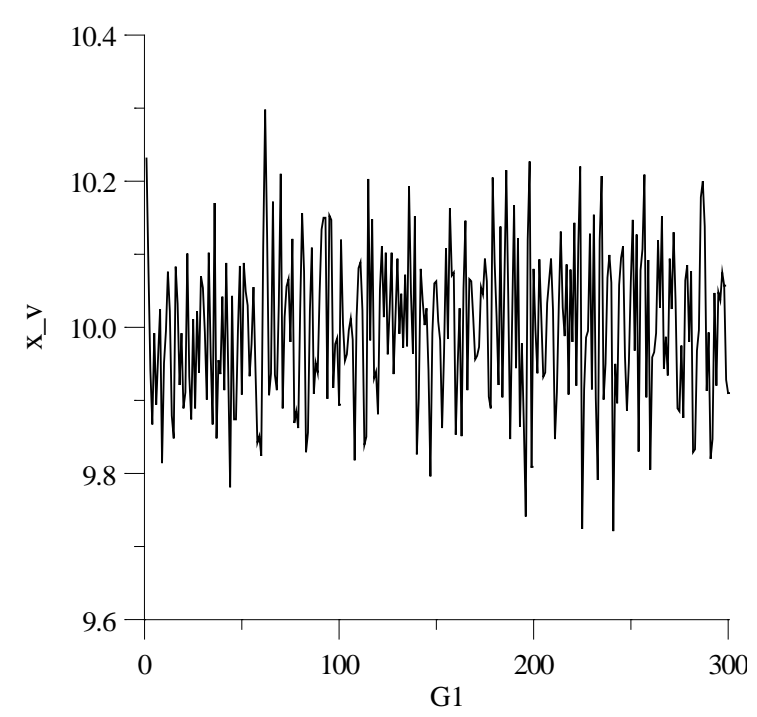

b)

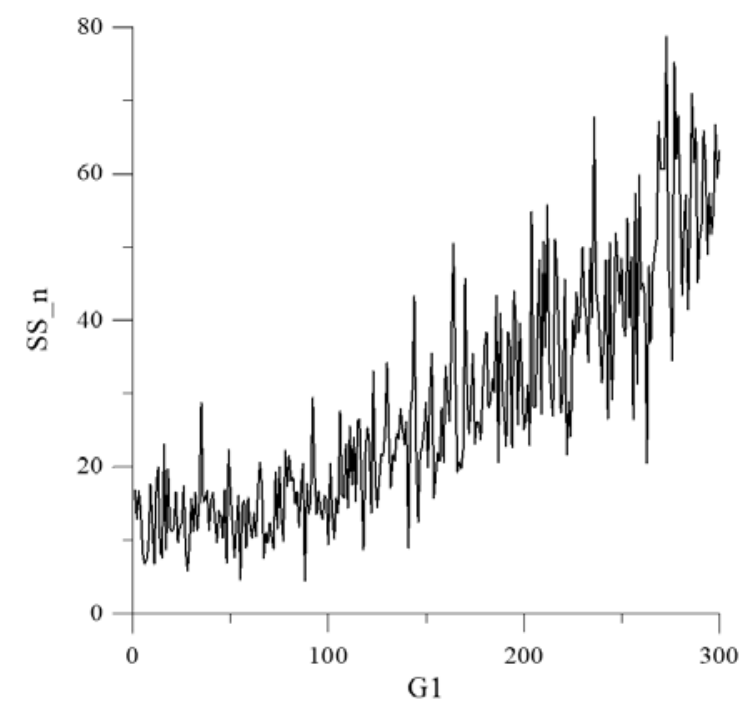

c)

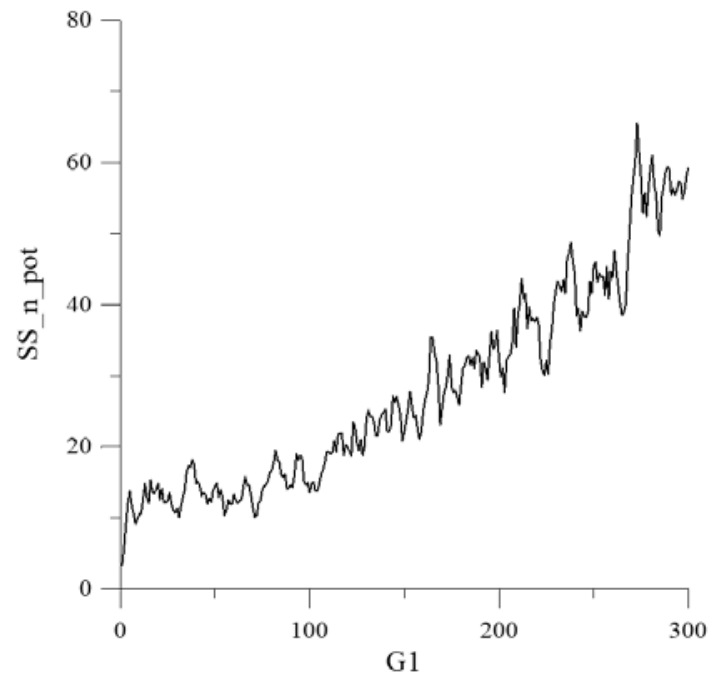

d)

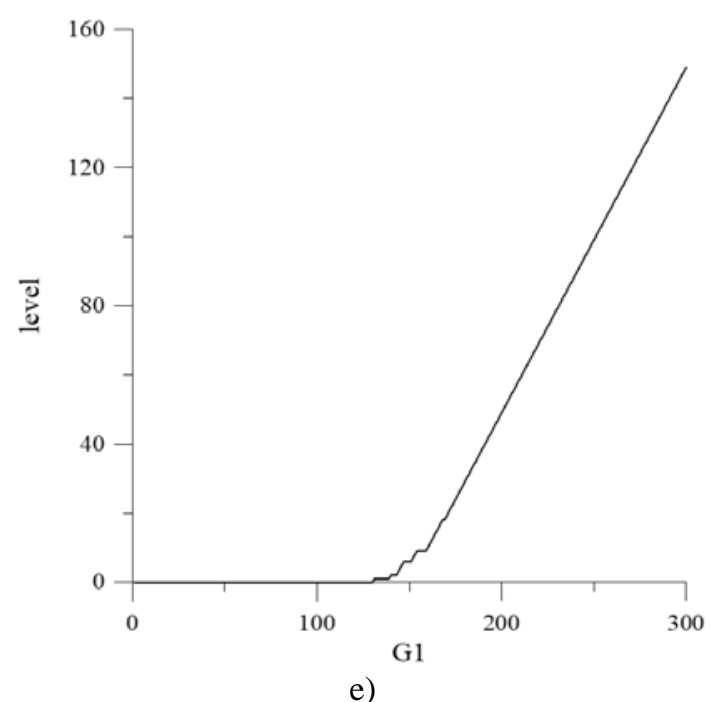

Fig.7. PPSG Statistic characteristics at fixed $G_{2}=0$ : (a) dependency $i_{\text {max }}$ on $G_{1}$, (b) - dependency $k_{b}$ (x_v) on $G_{1}$, (c) dependency $\chi_{c}^{2}$ (SS_n) on $G_{1}$, (d) - dependency $\chi_{c \text { p }}^{2}$ (SS_n_pot) on $G_{1}$, (e) - dependency $K_{L}$ (level) on $G_{1}$.

Further testing of the hypothesis occurs in this way.

1. According to the empirical distribution obtained as a result of the modeling of the PPSG operation, an average mean of values $k_{1}, k_{2}, \ldots, k_{n_{\max }}-k_{b}$ can be found.

2. Take as a parameter $\lambda$ of Poisson distribution a sample mean $-\lambda=k_{6}$.

3. Find, using Poisson's formula

$$
P_{j}=\lambda^{j} \frac{e^{-\lambda}}{j !}=k_{b}{ }^{j} \frac{e^{-k_{b}}}{j !}
$$


the probabilities of the equal $j$ pulses appearance (on interval $i_{\max }$ ) in $n_{\max }$ trials $(j=0,1,2, \ldots)$.

4. Find theoretical frequencies

$$
Q_{j}=P_{j} \cdot n_{\max }
$$

5. In the simulation process empirical frequencies are found $-N_{j}$.

6. For each value $j$ using the Pearson criterion are defined

$$
\begin{gathered}
S_{j}=\frac{\left(N_{j}-Q_{j}\right)^{2}}{Q_{j}} \\
\chi_{c}^{2}=\sum_{j=0}^{j_{\max }} S_{j}
\end{gathered}
$$

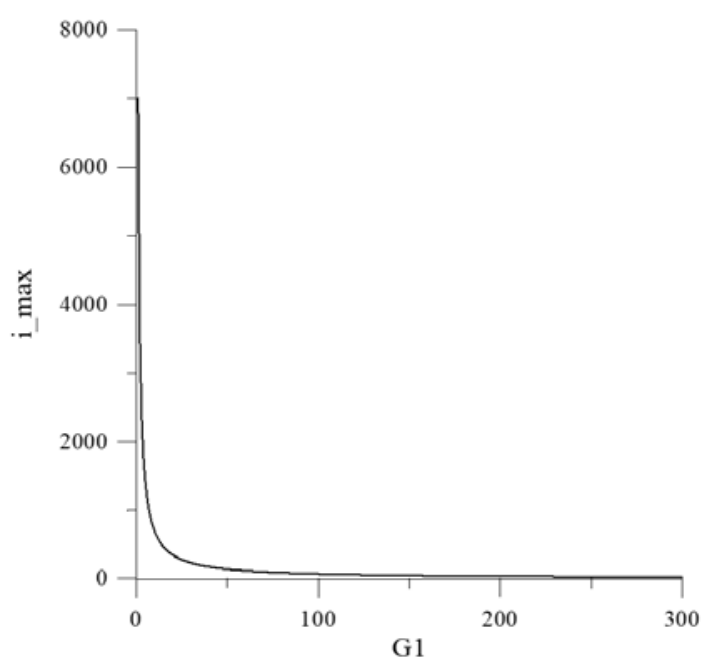

a)

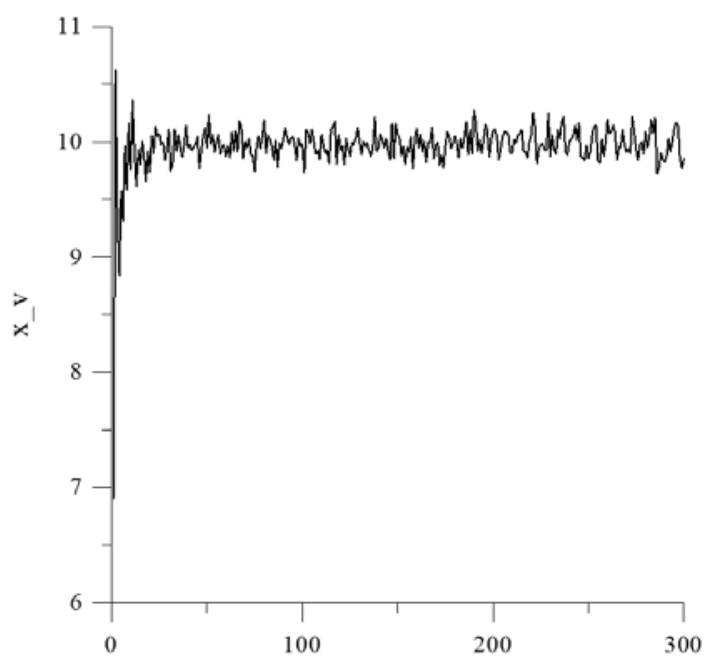

b)

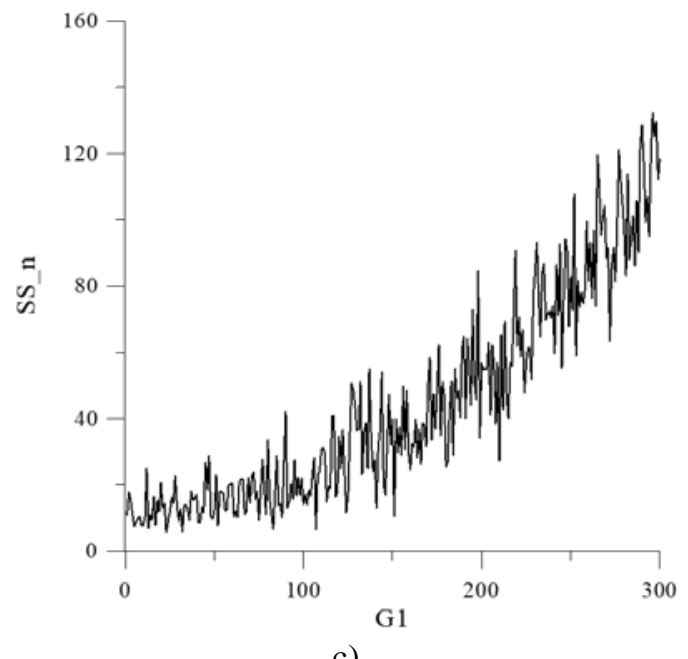

C)

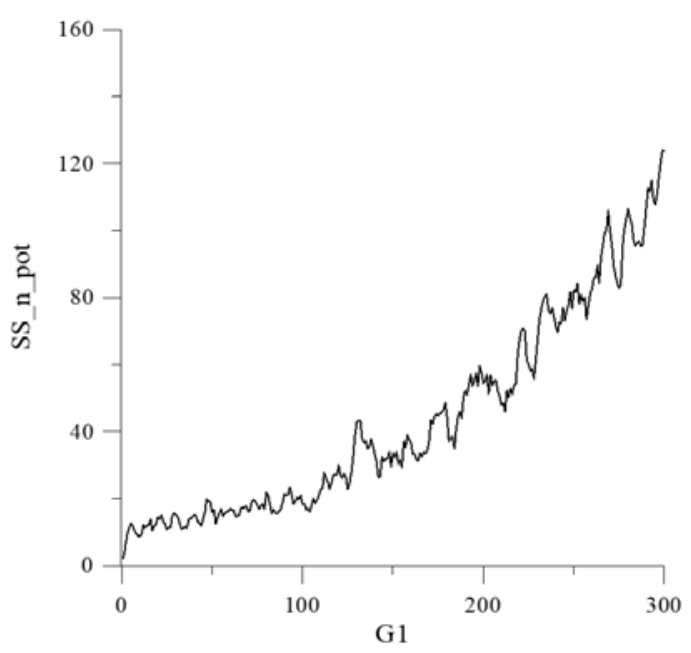

d)

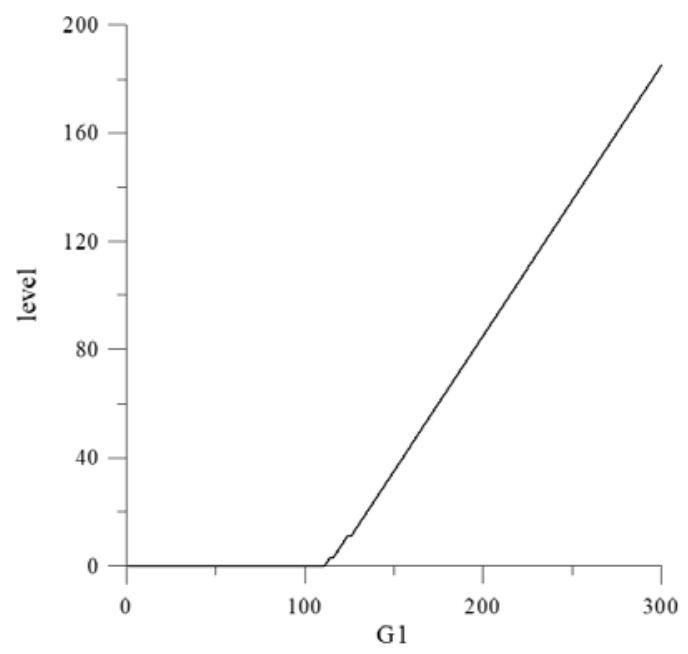

e)

Fig.8. PPSG Statistic characteristics at fixed $G_{2}=300$ : (a) dependency $i_{\max }$ on $G_{1}$, (b) - dependency $k_{s}$ (x_v) on $G_{1}$, (c) - dependency $\chi_{c}^{2}\left(\mathrm{SS} \_\mathrm{n}\right)$ on $G_{1}$, (d) - dependency $\chi_{c p}^{2}$ (SS_n_pot) on $G_{1}$, (e) - dependency $K_{L}$ (level) on $G_{1}$. 
7. If necessary values $N_{j}, P_{j}$, and $Q_{j}$ that correspond to probabilities $P_{j}$ are combined in one or two groups, and calculations (12) (13) are carried out on the basis of this fact.

8. The number of degrees of freedom is determined as follows

$$
r=d-2
$$

where $d$ - the number of groups remaining after the possible integration.

According to the tables of critical values of the distribution $\chi^{2}$ [36], for the selected level of significance $\alpha$ (usually $\alpha$ one of the three values $-0.1 ; 0.05 ; 0.01$ ) and the number of degrees of freedom $r$, the critical value $\chi_{\kappa p}^{2}$ is found. If $\chi_{c}^{2}<\chi_{k p}^{2}-$ the null hypothesis, that the pulse sequence is distributed according to the Poisson probability distribution, is accepted.

For the convenience of evaluating (defining) the range of control code values $G_{1}$ and $G_{2}$, for which the generator output sequence corresponds to the Poisson probability distribution, taking into account the probabilistic nature of the process, we additionally introduced the value $\chi_{c p}^{2}$ - the current average mean of the last five values $\chi_{c}^{2}$, as well as the value $K_{L}$ - the number of values $\chi_{c p}^{2}$ greater than $\chi_{\kappa p}^{2}$.

In Fig.7. and Fig.8. the results of research of the output signal of the PPSG (Fig.4.) are presented using the proposed method - in Fig.7. for $G_{2}=0$, and in Fig.8. for $G_{2}=300$.

Just as the results are presented in Fig.5., the dependences are obtained as a result of simulation for the program implementation of the PPSG, including the software implementation of the PRNG using the function random (it is for Delphi), at $D_{\max }=1000$ and clock speed fc $=1 \mathrm{kHz}$. It was set: $n_{\max }=1000, k_{c}=10, \chi_{k p}^{2}=25$. In Fig.7. and Fig.8. graphs of values $i_{\max }, k_{B}\left(\mathrm{x} \_\mathrm{v}\right), \chi_{c}^{2}\left(\mathrm{SS} \_\mathrm{n}\right), \chi_{c \mathrm{p}}^{2}$ (SS_n_pot), and $K_{L}$ (level) are shown, depending on the control code $G_{1}$.

The presented results allow us to determine the range of the control code values $G_{1}$, in which the output signal of the generator corresponds to the Poisson probability distribution. When $G_{2}=0$ this range is approximately $0 \div 125$, and at $G_{2}=300-0 \div 110$.

\section{CONCLUSIONS}

The analysis of the structures of the two types of digital devices was carried out in this paper, such as the two-level frequency synthesizer and the Poisson pulse sequence generator. The similarity of approaches to their construction allows creating a new type of devices that combine the useful properties of two previous ones - the ability to control the step of the output frequency changing and the formation of output pulse sequences in accordance with the Poisson probability distribution.

The new structure of the Poisson pulse sequence generator is presented, statistical characteristics of its output signal have been researched.

The proposed method for evaluating the statistical characteristics of the synthesizer output signal provides determination of its parameters, in which the output pulse flow corresponds to the Poisson probability distribution.

The proposed generators can be effectively used to simulate various natural and technical processes, in particular, to simulate the output signals of dosimetric detectors during the design, adjustment and testing of dosimetric devices.

Further researches in this direction will be, in particular, directed to optimizing the hardware implementation of synthesizers based on programmable logic integrated circuits.

\section{REFERENCES}

[1] Webster, J.G. (1998). The Measurement, Instrumentation and Sensors Handbook. CRC Press.

[2] Northrop, R.B. (2018). Introduction to Instrumentation and Measurements. CRC Press.

[3] Glowacz, A. (2019). Fault diagnosis of single-phase induction motor based on acoustic signals. Mechanical Systems and Signal Processing, 117, 65-80.

[4] Zhang, Y., Chen, B., Pan, G., Zhao, Y. (2019). A novel hybrid model based on VMD-WT and PCABPRBF neural network for short-term wind speed forecasting. Energy Conversion and Management, 195, 180-197.

[5] Przystupa, K. (2019). The methods analysis of hazards and product defects in food processing. Czech Journal of Food Sciences, 37 (1), 44-50.

[6] Michalowska, J., Tofil, A., Józwik, J., Pytka, J., Budzyński, P., Korzeniewska, E. (2018). Measurement of high-frequency electromagnetic fields in CNC machine tools area. In 2018 IEEE 4th International Symposium on Wireless Systems within the International Conferences on Intelligent Data Acquisition and Advanced Computing Systems (IDAACS-SWS). IEEE, p. 162-165.

[7] Birch, J.A. (2003). Benefit of Legal Metrology for the Economy and Society. A study for the International Committee of Legal Metrology. http://www.oiml. org/publications/E/birch/E002-e03.pdf

[8] Birch, J. (2012). Role and impact of legal metrology for consumer protection. In How do Consumers know what they are Getting? ISO/COPOLCO Workshop.

[9] Trisna, B.A., Rahman, A., Nugraha, A.R., Darmayanti, N.T.E., Pusaka, J. (2018). Current situation and prospect of nanometrology and its standardization in Indonesia. MAPAN Journal of Metrology Society of India, 33 (4), 469-480. 
[10] Wang, J., Kochan, O., Przystupa, K., Su, J. (2019). Information-measuring system to study the thermocouple with controlled temperature field. Measurement Science Review, 19 (4), 161-169.

[11] Przystupa, K. (2018). Reliability assessment method of device under incomplete observation of failure. In 2018 18th International Conference on Mechatronics Mechatronika (ME). IEEE, 1-6.

[12] Jun, S., Kochan, O., Chunzhi, W., Kochan, R. (2015). Theoretical and experimental research of error of method of thermocouple with controlled profile of temperature field. Measurement Science Review, 15 (6), 304-312.

[13] Pavlasek, P., Rybař, J., Ďuriš, S., Palenčar, J. (2019). Effects of quartz glass insulation on platinum gold thermocouples. Measurement Science Review, 19 (5), 209-212.

[14] Jun, S., Kochan, O., Kochan, R. (2016). Thermocouples with built-in self-testing. International Journal of Thermophysics, 37 (4), 37.

[15] Wojciechowski, S., Wiackiewicz, M., Krolczyk, G.M. (2018). Study on metrological relations between instant tool displacements and surface roughness during precise ball end milling. Measurement, 129, 686-694.

[16] Shu, C., Kochan, O. (2013). Method of thermocouples self verification on operation place. Sensors \& Transducers, 160 (12), 55-61.

[17] Mitryasova, O., Pohrebennyk, V., Kochanek, A., Sopilnyak, I. (2016). Correlation interaction between electrical conductivity and nitrate content in natural waters of small rivers. In 16th International Multidisciplinary Scientific GeoConference SGEM 2016, 3 (3), 357-364.

[18] Wang, T.P., Bediones, D.P., Henrikson, H.J., Janhunen, E.J., Bachalo, K., Swirla, P. (1997). Stabilized metal sheathed type K and E thermocouples improve turbine efficiency. In ISA TECH/EXPO Technology Update, 1 (5), 439-448.

[19] Glowacz, A. (2018). Recognition of acoustic signals of commutator motors. Applied Sciences, 8 (12), 2630.

[20] Pelc, M., Khoma, Y., Khoma, V. (2019). ECG signal as robust and reliable biometric marker: Datasets and algorithms comparison. Sensors, 19 (10), 2350.

[21] Kroupa, V.F. (1999). A Digital Frequency Synthesizer. Wiley-IEEE Press.

[22] Vankka, J., Halonen, K.A. (2013). Direct Digital Synthesizers: Theory, Design and Applications. Springer.

[23] Chenakin, A. (2011). Frequency Synthesizers: Concept to Product. Artech House.

[24] Maksymovych, V., Stakhiv, R. (2002). Two-level frequency synthesizer. Bulletin of the Lviv Polytechnic National University: Computer Engineering and Information Technologies, 468, 29-34. (in Ukrainian)
[25] Maksymovych, V., Stakhiv, R. (2003). Two-level frequency synthesizer error analysis. Bulletin of the Lviv Polytechnic National University: Computer Engineering and Information Technologies, 496, 1722. (in Ukrainian)

[26] Arkani, M., Khalafi, H., Vosoughi, N. (2013). A flexible multichannel digital random pulse generator based on FPGA. World Journal of Nuclear Science and Technology, 3 (4), 109-116.

[27] Veiga, A., Spinelli, E. (2016). A pulse generator with poisson-exponential distribution for emulation of radioactive decay events. In 2016 IEEE 7th Latin American Symposium on Circuits \& Systems (LASCAS). IEEE, 31-34.

[28] Heeger, D. (2000). Poisson model of spike generation. Handout, University of Standford, 5, 1-13.

[29] Garasymchuk, O., Dudykevych, V., Maksymovych, V., Smuk, R. (2004). Generators of test pulse sequences for dosimetric devices. Bulletin of the Lviv Polytechnic National University: Heat Power Engineering. Environmental Engineering. Automation, 506, 187-193. (in Ukrainian)

[30] Maksymovych, V., Stakhiv, R., Stakhiv, M. (2019). The modified structure of the two-level digital frequency synthesizer for dosimetry. Measuring Equipment and Metrology, 80 (1), 17-20. doi.org/10.23939/istcmtm2019.01.017. (in Ukrainian)

[31] Maksymovych, V., Stakhiv, R. (2005). Two-level digital synthesizer on the basis on two combinational adders with eliminating of the uneven output pulses. Collection of Scientific Works of the Ukrainian Academy of Printing: Computer Technologies of Printing, 13, 227-234. (in Ukrainian)

[32] Bobalo, Yu., Dudykevych, V., Maksymovych, V., Khoroshko, V., Bisyk, A., Smuk, R., Storonskyi, Yu. (2009). Methods and Means of Working Out the Output Signals of the Dosimetric Detectors. Publishing House of Lviv Polytechnic National University. (in Ukrainian)

[33] Kostiv, Yu., Maksymovych, V., Harasymchuk, O., Mandrona, M. (2013). Methodology for research of poisson pulse sequence generators using Pearsons' chi-squared test. Sustainable Development: International Journal, 9, 67-72.

[34] Schneier, B. (2007). Applied Cryptography: Protocols, Algorithms, and Source Code in C. John Wiley \& Sons.

[35] Buchmann, J. (2013). Introduction to Cryptography. Springer.

[36] DeCoursey, W. (2003). Statistics and Probability for Engineering Applications. Elsevier.

Received November 4, 2019 Accepted March 10, 2020 